\title{
Vessel-dependent recruitment of sympathetic axons: looking for innervation in all the right places
}

\author{
Yoh-suke Mukouyama \\ Laboratory of Stem Cell and Neuro-Vascular Biology, Genetics and Developmental Biology Center, \\ National Heart, Lung, and Blood Institute, NIH, Bethesda, Maryland, USA.
}

\begin{abstract}
Autonomic sympathetic axons extend along and innervate resistance arteries to control vascular tone and participate in blood pressure regulation. In this issue of the JCI, Brunet and colleagues reveal that sympathetic innervation of arteries is facilitated by secretion of the axon guidance molecule netrin-1 by arterial VSMCs. Furthermore, disruption of the signaling cascade induced by netrin-1 through its receptor DCC resulted in defective arterial innervation and sympathetic control of vasoconstriction. This comprehensive study represents a major step forward in our understanding of the coordinated wiring of the vascular and nervous systems in various tissues.
\end{abstract}

\section{Coordinated branching morphogenesis of blood vessels and nerves}

Branching morphogenesis is a fundamental attribute of many organ systems. As examples of highly informative model systems for branching morphogenesis, the vascular and nervous systems share several anatomical and functional characteristics and are often patterned similarly in peripheral tissues. Specialized cells located at the extremities of capillaries, referred to as endothelial tip cells, are highly similar to growth cones of developing axons (1). Furthermore, several studies have revealed that guidance molecules, such as netrins, semaphorins, ephrins, and slits, that are essential for neuronal axon branching are also important for the branching of growing blood vessels (2).

As evidenced by the alignment of their branching patterns in many tissues, branching morphogenesis and patterning of both blood vessel and nerves need to be integrated (3). In developing skin, sensory nerves can provide a unique anatomical template that determines arterial branching patterns (4). Nerve-derived VEGF-A controls arterial differentiation $(4,5)$, and nerve-derived CXCL12 directs vessel branching and alignment with nerves (6). Conversely, blood vessel-derived cues, such as artemin (7), neurotrophin-3 (NT-3)

Conflict of interest: The author has declared that no conflict of interest exists.

Citation for this article: J Clin Invest. 2014; 124(7):2855-2857. doi:10.1172/JCI76622.
$(8,9)$, nerve growth factor (NGF) (10), and endothelins (ETs) (11), attract growing sympathetic axons to travel alongside blood vessels. Together, these findings suggest that either blood vessels or nerves develop branches and then instruct the other branches through the expression and secretion of growth cues from the already established branches in order to develop in alignment alongside each other, raising the intriguing question of how the neurovascular interactions and copatterning influence organ physiology, regeneration, and disease.

\section{What controls sympathetic innervation of arteries?} eter blood vessels covered with VSMCs to control vascular tone and participate in blood pressure regulation. In parallel with vascular innervation, axons extend along large-diameter vessels to reach and innervate target organs. While vascular-derived signals, such as artemin, NT-3, NGF, and ETs, mainly guide sympathetic axon extension along blood vessels, the process that determines the innervation of blood vessel themselves is poorly understood. In this study, Brunet and colleagues identified the axon guidance molecule netrin-1 as a critical arterial VSMC-secreted factor for facilitating the sympathetic innervation of arteries (12). Specifically, Brunet et al. revealed that arterial VSMCs express netrin- 1 at the onset of innervation, while the netrin-1 receptor deleted in colorectal cancer (DCC) is expressed by sympathetic
Sympathetic axons innervate large-diam- axons. Genetic alterations of netrin- $1 /$ DCC signaling lead to significant reduction $(-50 \%)$ in sympathetic innervation of arteries, including mesenteric and skin arteries. Indeed, mice with a VSMC-specific deletion of the gene encoding netrin-1 essentially phenocopied mice with a sympathetic neuron-specific deletion of $D c c$. Furthermore, heterozygous netrin-1 mutants also exhibited impaired arterial innervation in the skin, allowing exploration of local vasoconstriction within these mice. The heterozygous mutants displayed less vasoconstriction compared with control animals in response to a drop of core body temperature. Together, these findings strongly suggest that arterial VSMC-derived netrin-1 promotes sympathetic innervation of arteries via axonal DCC and that the reduced arterial innervation due to deficient netrin-1/DCC signaling leads to defective vasoconstriction in the arteries.

\section{Does netrin-1/DCC signaling induce specific sympathetic axons to innervate arteries?}

Sympathetic axons generate branches to connect with multiple targets. There has been ongoing debate about how the location of sympathetic axon branching is specified. Sympathetic innervation of mesenteric arteries develops after the axons have connected with the gut (Figure 1). Interestingly, the mesenteric arteries do not express netrin-1 during embryonic developmental stages, when axons are navigating along the arteries to the gut (Figure 1A). After birth, netrin-1 is present in the mesenteric arteries and stimulates the formation of interstitial branches to innervate the arteries (Figure 1B). This temporal control of netrin-1 production results in the axons innervating both the gut and mesenteric arteries, yet the spatiotemporal regulation of netrin-1 in this process remains to be elucidated. In support of this observation, previous studies demonstrated that netrin- 1 can 

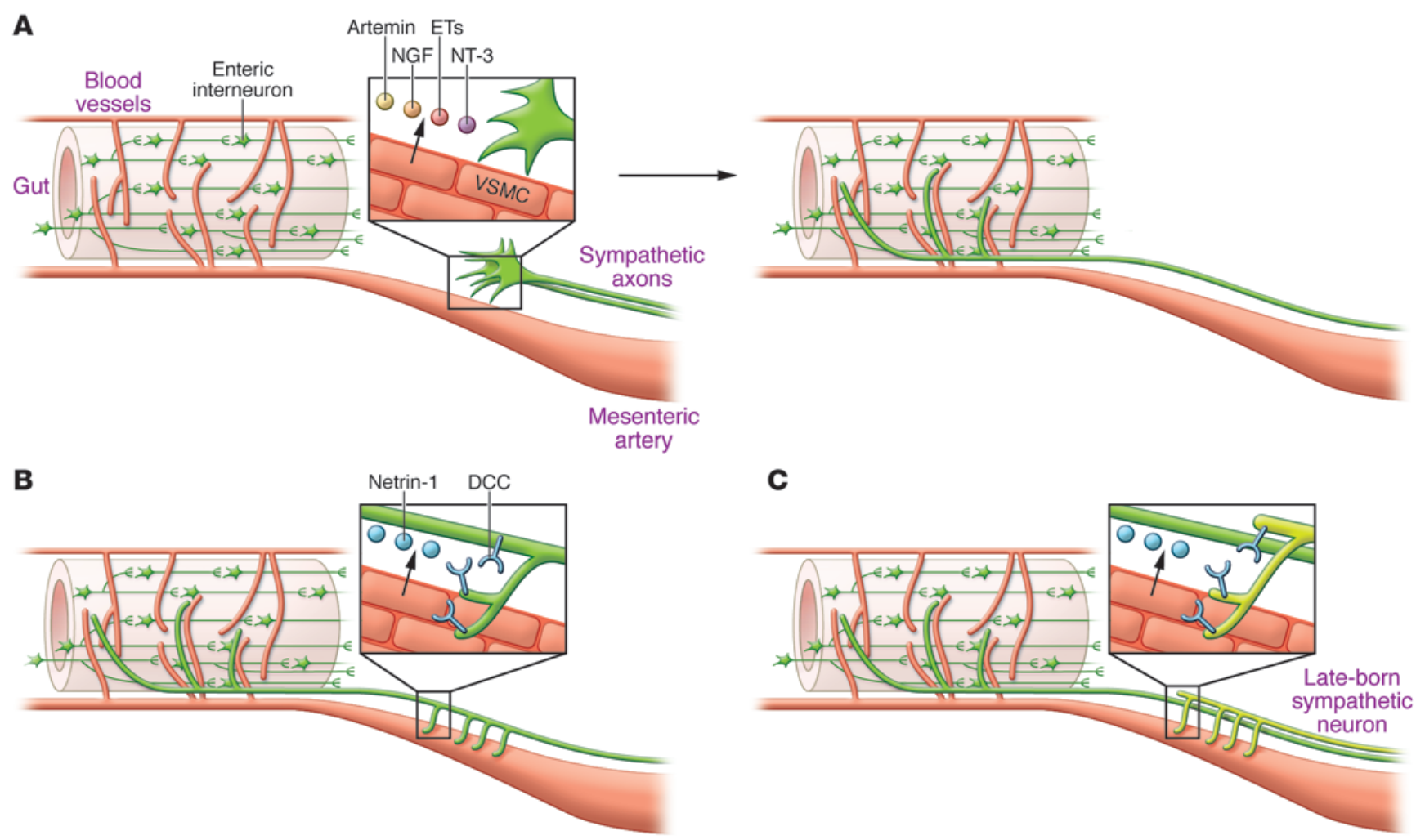

\section{Figure 1}

Sympathetic innervation of the gut and mesenteric arteries. (A) Vascular-derived signals, such as artemin, NT-3, NGF, and ETs, guide sympathetic axon extension along mesenteric arteries. Sympathetic innervation of mesenteric arteries develops after the axons have connected with the gut and the alignment of sympathetic nerves and mesenteric arteries has been established. There are two possible models to explain how sympathetic axons project both to the gut and mesenteric arteries, as illustrated in B and C. (B) The "interstitial branches for innervation" model suggests that VSMC-derived netrin-1 triggers established sympathetic nerves to form interstitial branches for arterial innervation. (C) The "lateborn neurons for innervation" model suggests that there are two distinct sets of sympathetic axons that project either to the gut or the mesenteric arteries, and the axons that selectively project to the mesenteric arteries develop at a relatively late embryonic stage compared with the axons that project to the gut.

stimulate branch formation of cultured cortical axons when applied locally (13). Given that the mesenteric arteries are innervated by a subset of DCC-expressing axons, some axons generate branches to innervate the arteries in response to arterial netrin-1, while others do not generate any branches. This could be explained by the presence of an underlying mechanism that involves coordination of repulsive signals, such as semaphorins and ephrins, with netrin-1 to restrict a subset of sympathetic axon branching and innervation to the arteries.

An alternative model is that there are two distinct sets of sympathetic axons that project either to the gut or the mesenteric arteries. This would require axons that project selectively to the mesenteric arteries to develop at a relatively late embryonic stage compared with the axons that project to the gut (Figure 1C). It is clear that target-derived signals promote innervation such that a subset of sympathetic axons innervates the mesenteric arteries in response to VSMC-derived netrin-1. Whether such subpopulations exhibit different molecular characteristics and require different target-derived factors for survival, differentiation, and innervation is an intriguing question. Given that sympathetic neuron subtypes express unique combinations of neurotransmitters, neuropeptides, ion channels, and receptors, which together comprise the chemical phenotype of the neurons, there are two proposed models for the generation of the diversity of sympathetic neuron subtypes during embryonic and early postnatal development. One model is that each neuronal subtype is predetermined at an early stage of development and adopts a particular chemical phenotype that promotes exten- sion of its axon to the matching target. The alternative model posits that neurons randomly extend to contact targets, and signals from the target tissues they innervate induce neurons to adopt the appropriate chemical phenotype (14-16).

Of note, vagal sensory axons that carry signals from the bowel to the brain also project to the gut at an early embryonic stage (E12 E14) (17). Because some vagal sensory axons express tyrosine hydroxylase $(\mathrm{TH})$, which catalyzes the rate-limiting step in catecholamine biosynthesis and is used as a suitable marker for sympathetic axons, use of the anti-TH antibody for immunohistochemical evaluation may not accurately distinguish between vagal sensory axons and sympathetic axons in the gut (18). Furthermore, netrin-1 expression is detectable in the outer gut mesenchyme and mucosal epithelium and attracts DCC-expressing 
vagal sensory axons to the gut (19). It is not clear whether vagal sensory axons also extend along the mesenteric arteries to connect with the gut, but future efforts should focus on the establishment of an optical map of the physical patterns of innervation for different axon subtypes and the required guidance signals, such as netrin-1, to direct these patterns for different types of axons.

\section{Concluding remarks}

The alignment between blood vessel and nerve branching ensures that these two systems communicate within integrated tissues. Arteries supply oxygen and nutrients for nerves, while nerves control vascular tone and blood pressure through their vascular innervation. Emerging studies are beginning to reveal important information on how this relationship develops. The work of Brunet and colleagues clearly demonstrates that the interaction between netrin-1, which is released by arterial VSMCs, and DCC, which is expressed on sympathetic axons, underlies the proper formation of axonal branches and subsequent arterial innervation, a critical step in the establishment of vascular physiology. Several important questions regarding the relationship between the netrin-1/DCC pathway and innervation remain. In addition to directing innervation, does netrin-1/DCC signaling also influence the maintenance of innervation? Do other VSMC-derived axon guidance signals control vascular innervation in other tissues? How do VSMCderived signals coordinate axon extension and innervation? Future investigation of these questions will enhance our understanding of the coordinated wiring of the vascular and nervous systems.

\section{Acknowledgments}

I thank R.S. Adelstein and K. Gill for editorial advice on the manuscript and J. Hatch for invaluable discussion. Studies cited in the reference list by the author's laboratory were supported by the Intramural Research Program of the National Heart, Lung, and Blood Institute, NIH (HL005702 and HL006116).

Address correspondence to: Yoh-suke Mukouyama, Laboratory of Stem Cell and Neuro-Vascular Biology, Genetics and Developmental Biology Center, National Heart, Lung, and Blood Institute, National Institutes of Health, Building 10/6C103, 10 Center Drive, Bethesda, Maryland 20892, USA. Phone: 301.451.1663; Fax: 301.480.1581; E-mail: mukoyamay@mail.nih.gov.

1. Gerhardt H, et al. VEGF guides angiogenic sprouting utilizing endothelial tip cell filopodia.J Cell Biol. 2003;161(6):1163-1177

2. Adams RH, Eichmann A. Axon guidance molecules in vascular patterning. Cold Spring Harb Perspect Biol. 2010;2(5):a001875.

3. Lu P, Werb Z. Patterning mechanisms of branched organs. Science. 2008;322(5907):1506-1509.

4. Mukouyama YS, Shin D, Britsch S, Taniguchi M, Anderson DJ. Sensory nerves determine the pattern of arterial differentiation and blood vessel branching in the skin. Cell. 2002;109(6):693-705.

5. Mukouyama YS, Gerber HP, Ferrara N, Gu C, Anderson DJ. Peripheral nerve-derived VEGF promotes arterial differentiation via neuropilin 1mediated positive feedback. Development. 2005; 132(5):941-952.
6. Li W, et al. Peripheral nerve-derived CXCL12 and VEGF-A regulate the patterning of arterial vessel branching in developing limb skin. Dev Cell. 2013; 24(4):359-371.

7. Honma Y, et al. Artemin is a vascular-derived neurotropic factor for developing sympathetic neurons. Neuron. 2002;35(2):267-282.

8. Francis N, et al. NT-3, like NGF, is required for survival of sympathetic neurons, but not their precursors. Dev Biol. 1999;210(2):411-427.

9. Kuruvilla R, et al. A neurotrophin signaling cascade coordinates sympathetic neuron development through differential control of TrkA trafficking and retrograde signaling. Cell. 2004;118(2):243-255.

10. Nam J, et al. Coronary veins determine the pattern of sympathetic innervation in the developing heart. Development. 2013;140(7):1475-1485.

11. Makita T, Sucov HM, Gariepy CE, Yanagisawa M, Ginty DD. Endothelins are vascular-derived axonal guidance cues for developing sympathetic neurons. Nature. 2008;452(7188):759-763.

12. Brunet I, et al. Netrin-1 controls sympathetic arterial innervation. J Clin Invest. 2014;124(7):3230-3240.

13. Dent EW, Barnes AM, Tang F, Kalil K. Netrin-1 and semaphorin $3 \mathrm{~A}$ promote or inhibit cortical axon branching, respectively, by reorganization of the cytoskeleton. J Neurosci. 2004;24(12):3002-3012.

14. Glebova NO, Ginty DD. Growth and survival signals controlling sympathetic nervous system development. Annu Rev Neurosci. 2005;28:191-222.

15. Cane KN, Anderson CR. Generating diversity: Mechanisms regulating the differentiation of autonomic neuron phenotypes. Auton Neurosci. 2009; 151(1):17-29.

16. Rohrer H. Transcriptional control of differentiation and neurogenesis in autonomic ganglia. Eur J Neurosci. 2011;34(10):1563-1573.

17. Ratcliffe EM, Farrar NR, Fox EA. Development of the vagal innervation of the gut: steering the wandering nerve. Neurogastroenterol Motil. 2011; 23(10):898-911.

18. Kummer W, Bachmann S, Neuhuber WL, Hanze J, Lang RE. Tyrosine-hydroxylase-containing vagal afferent neurons in the rat nodose ganglion are independent from neuropeptide-Y-containing populations and project to esophagus and stomach. Cell Tissue Res. 1993;271(1):135-144.

19. Ratcliffe EM, Setru SU, Chen JJ, Li ZS, D'Autreaux F, Gershon MD. Netrin/DCC-mediated attraction of vagal sensory axons to the fetal mouse gut. J Comp Neurol. 2006;498(5):567-580. 